\title{
Heating and compression of a laser produced plasma in a pulsed magnetic field
}

J. R. Creel, T. Donnelly, and J. G. Lunney

Citation: Appl. Phys. Lett. 109, 071104 (2016); doi: 10.1063/1.4961115

View online: http://dx.doi.org/10.1063/1.4961115

View Table of Contents: http://aip.scitation.org/toc/apl/109/7

Published by the American Institute of Physics

\section{Articles you may be interested in}

Spatially resolved behavior of laser-produced copper plasma along expansion direction in the presence of static uniform magnetic field

Appl. Phys. Lett. 23, 122104122104 (2016); 10.1063/1.4969080

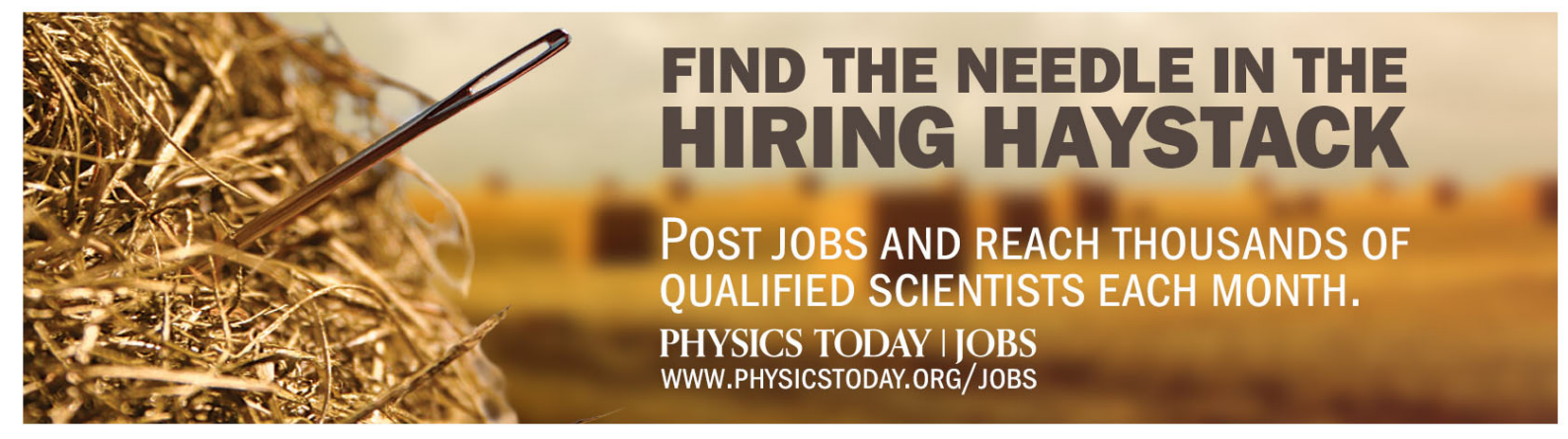




\title{
Heating and compression of a laser produced plasma in a pulsed magnetic field
}

\author{
J. R. Creel, ${ }^{\text {a) }}$ T. Donnelly, and J. G. Lunney \\ School of Physics and CRANN, Trinity College Dublin, The University of Dublin, Dublin 2, Ireland
}

(Received 22 June 2016; accepted 4 August 2016; published online 15 August 2016)

\begin{abstract}
A pulsed $0.3 \mathrm{~T}$ magnetic field was used to heat and compress a low-temperature laser produced copper plasma. The magnetic field was generated using a planar 3-turn coil positioned $10 \mathrm{~mm}$ above the ablation spot. The plasma flowing through a central aperture in the coil was strongly focused. Inductive heating of the plasma caused a large enhancement of the overall visible light emission and the appearance of $\mathrm{Cu}$ II line emission. The plasma focusing is also evident in the constriction of the spatial distribution of deposited copper. The plasma heating and focusing can be explained in the framework of resistive magnetohydrodynamics. Published by AIP Publishing.

[http://dx.doi.org/10.1063/1.4961115]
\end{abstract}

There is continuing interest in using quasi-static magnetic fields for the manipulation and excitation of laser produced plasma (LPP), both in terms of understanding the magnetohydrodynamic (MHD) interaction and enhancing applications such as pulsed laser deposition (PLD) and laser induced breakdown spectroscopy (LIBS). In PLD, particulate contamination on substrates can be avoided by using a magnetic field to guide a plasma ablation plume on a curved path. ${ }^{1}$ Placing a permanent magnet behind the deposition substrate leads to an increased deposition rate and improved film quality in PLD of $\mathrm{SrTiO}_{3}{ }^{2}$ Pagano et al. ${ }^{3}$ have shown that a plasma lens placed between the target and substrate increases the deposition rate. There are several reports on using a magnetic field oriented parallel to the target surface to limit the forward expansion of the plasma plume and enhance ionization and optical emission. ${ }^{4-7}$ Magnetic confinement of LPP has been used to form an elongated plasma column for X-ray laser studies. ${ }^{8}{ }^{8}$ There is extensive literature on Z-pinch discharges in LPP for X-ray ${ }^{10}$ or extreme ultraviolet radiation sources. ${ }^{11,12}$

Here, we report on the inductive heating and magnetic focusing of a LPP during the first few microseconds of ablation plume expansion. Time-resolved imaging was used to capture the expansion dynamics, with and without the pulsed magnetic field. Plasma focusing was also observed via the distribution of the deposited material. Optical emission spectroscopy showed strong enhancement of the light emission and the emergence of $\mathrm{Cu}$ II emission due to inductive heating.

The experimental arrangement is shown in Fig. 1 where an induction coil is symmetrically positioned $10 \mathrm{~mm}$ above the laser ablation spot on a rotating copper $(\mathrm{Cu})$ target in a vacuum chamber at $10^{-5}$ mbar. Timing of the pulsed magnetic field was controlled with respect to the laser ablation pulse. The $\mathrm{Cu}$ target was ablated using a $248 \mathrm{~nm}, 20 \mathrm{~ns}$ laser at normal incidence at a fluence of $\sim 2 \mathrm{~J} \mathrm{~cm}^{-2}(\mathrm{E}=60 \mathrm{~mJ}$, circular area $=0.03 \mathrm{~cm}^{2}$ ). The pulsed magnetic field was produced using a 3-turn spirally wound planar induction coil. It was constructed using $1.1 \mathrm{~mm}$ diameter insulated copper

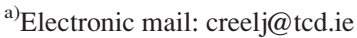

wire and set in a resin annulus with a central aperture of $10 \mathrm{~mm}$ and an outer diameter of $18 \mathrm{~mm}$ (Fig. 1(b)). The selfinductance $\left(\mathrm{L}_{1}\right)$ of the coil was calculated to be $280 \mathrm{nH}$. The coil was energized by the discharge of a $470 \mathrm{nF}$ capacitor (C) charged to $1.5 \mathrm{kV}$. The discharge was initiated at a controllable time with respect to the laser pulse using a silicon controlled rectifier (S). The rate of change of the current in the coil was measured using a Rogowski coil, and the coil current was obtained by integration.

A negatively biased $(-25 \mathrm{~V}) 0.04 \mathrm{~cm}^{2}$ planar Langmuir probe placed at $15 \mathrm{~cm}$ from the target was used to monitor the ion flux in the plasma flowing through the coil aperture. ${ }^{13}$ The visible light emission was recorded using a fast, intensified charge coupled device (ICCD) camera $(380-700 \mathrm{~nm}$ ) and with a time- and space-resolving spectrometer operating in the wavelength range of $488-532 \mathrm{~nm}$. Both the ICCD camera and the spectrometer used $200 \mathrm{~ns}$ gate widths triggered at a controllable delay after the laser pulse. To enhance the signal-to-noise ratio of the images and space-resolved spectra, an average of 50 acquisitions was used.

Figure 2(a) shows plots of the ion current density due to the plasma flow at the coil without magnetic field, the coil current, and a photodiode record of the laser pulse. The current density $j_{i}$ recorded by the ion probe at position $z_{p}$ is given by $j_{i}\left(z_{p}, t\right)=n_{i}\left(z_{p}, t\right) u e$, where $n_{i}\left(z_{p}, t\right)$ is the ion density at the probe, $u$ is the plasma flow velocity, and the ions are assumed to be singly charged. Thus, the ion probe measures the plasma velocity and density. Since beyond a few
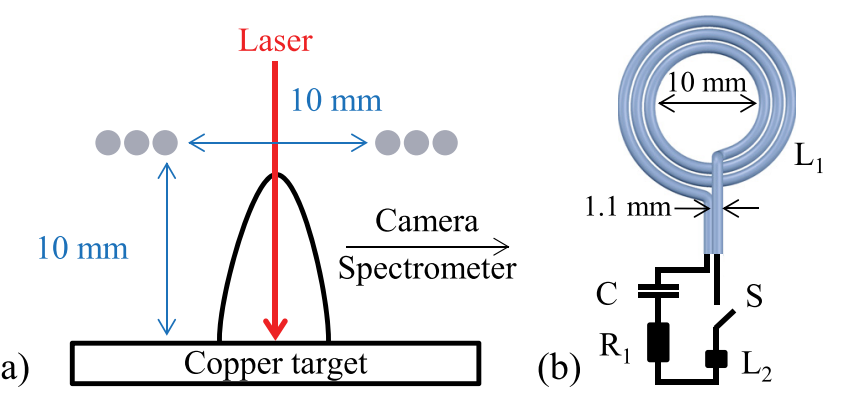

FIG. 1. (a) Side view of the experimental setup. (b) Planar induction coil with equivalent discharge circuit. 

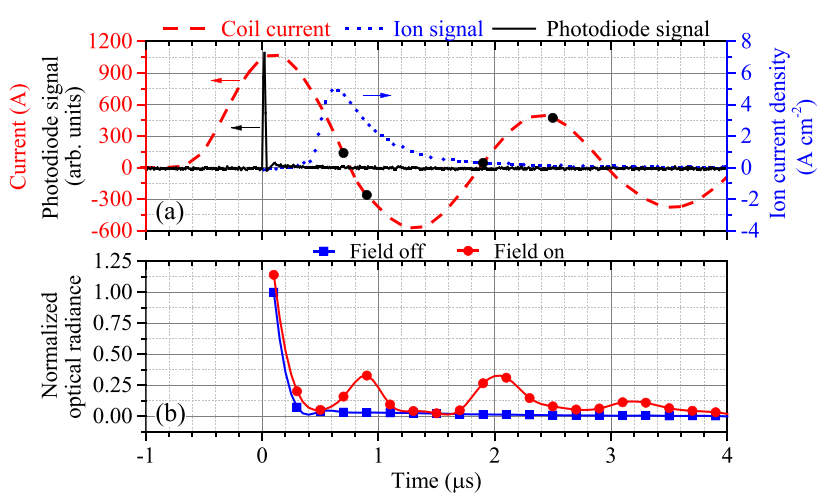

FIG. 2. (a) Plot of the current in the magnetic coil, the ion current density at the coil center, and the photodiode record of the laser. The black circles show the times of the plasma images shown in Fig. 3. (b) Optical radiance integrated over the whole plasma with field off and field on, normalized to field off case at $100 \mathrm{~ns}$.

millimeters of plume expansion, the plume is self-similar and inertial, the measured ion flux at the probe position $\left(z_{p}\right)$ can be used to calculate ion current density at the coil position. $^{14}$

The pulse frequency of the laser and magnetic field was $0.5 \mathrm{~Hz}$. The coil current was underdamped with a period of $2.2 \mu \mathrm{s}$. The current profile was fitted using values of $0.19 \Omega$ and $315 \mathrm{nH}$ for the overall resistance and inductance, respectively. For a charging voltage of $1.5 \mathrm{kV}$, the peak current was $1070 \mathrm{~A}$ corresponding to a magnetic field of $0.3 \mathrm{~T}$ at the center of the coil, as calculated using COMSOL Multiphysics. ${ }^{15}$ There was no noticeable difference in the coil current measured with, and without, the plasma plume, indicating relatively weak energy coupling to the plasma. The timing of the discharge was chosen such that the rate of change of magnetic field goes through a maximum at $700 \mathrm{~ns}$, where maximum ion flux at the coil is observed. Figure 2(b) shows the optical radiance integrated over the whole plasma with field off and field on, normalized to the field off case at $100 \mathrm{~ns}$. Due to the inductive plasma heating, optical emission is maximized at the same times as the maxima of rate of change of current.

Figure 3 shows a selected time sequence of ICCD images of the visible light emission with and without magnetic field. At $600 \mathrm{~ns}$, in the absence of the magnetic field, the ablation plume is approximately semi-ellipsoidal in shape, in agreement with the adiabatic and isentropic model of plume expansion. ${ }^{13,16}$ The leading edge of the plume has just reached the coil which is in accordance with the ion signal in Fig. 2(a). At later times, it can be seen that the $10 \mathrm{~mm}$ diameter coil aperture acts as a skimmer that transmits the central portion of the plume. The intensity of the visible light from the expanding plume falls steadily with time.

In the presence of the magnetic field, the ICCD images reveal more complex behavior, and at various times, the intensity of the light emission is much higher than for the no-field case. The timing of the magnetic field was chosen such that the ablation plume enters the coil at $600 \mathrm{~ns}$ when the rate of change of magnetic field is at a maximum. Thus, we conclude that the increased emission seen from 600 to $1000 \mathrm{~ns}$ is due to inductive heating of the plasma. At $900 \mathrm{~ns}$, the region of intense light emission on the target side of the coil can be understood as a side-on view of a ring of higher

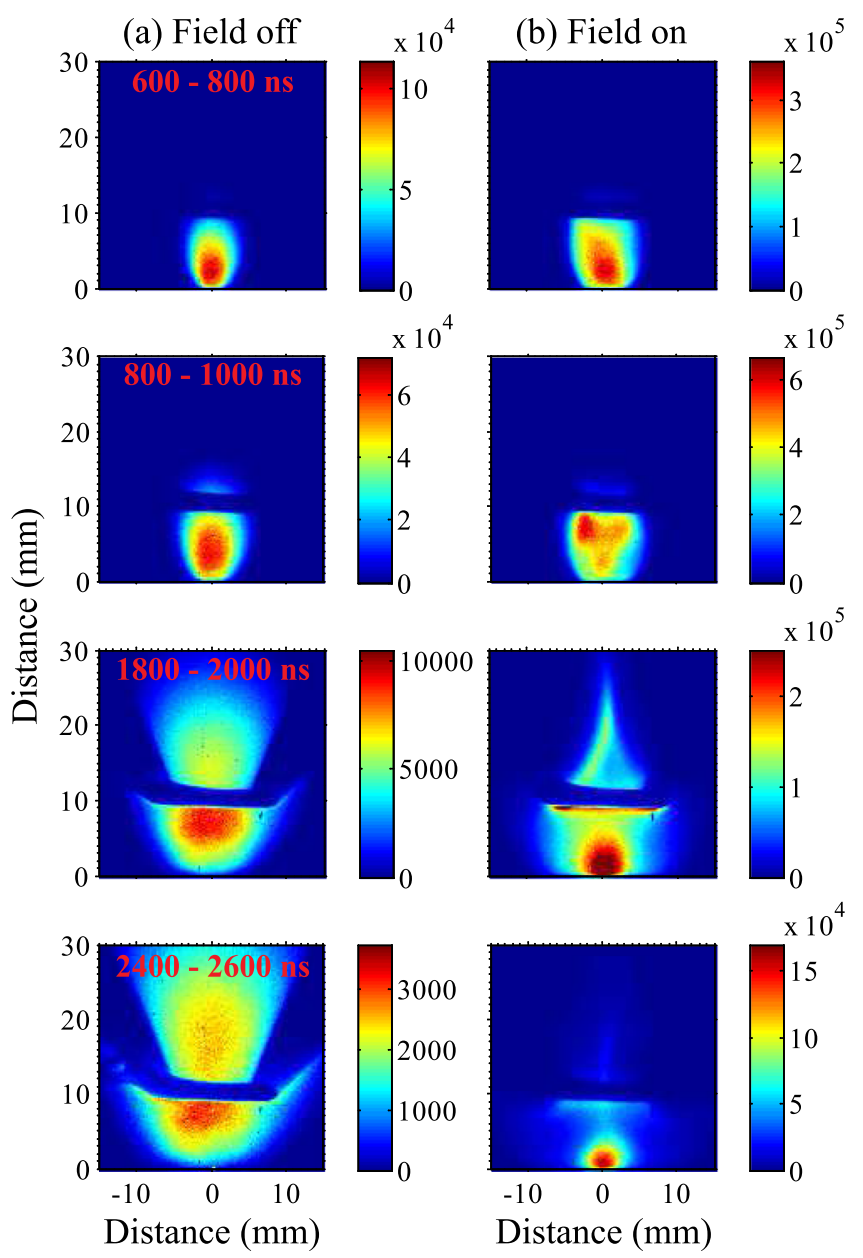

FIG. 3. ICCD imaging showing the plume (a) without and (b) with the magnetic field at various timings. Images are $30 \times 30 \mathrm{~mm}^{2}$ and the color bars indicate count numbers.

light emission excited by inductive heating near the coil where the magnetic field is strongest. Spectrally resolved imaging (not shown here) revealed that this light emission is mainly due to $\mathrm{Cu}$ II. At $1900 \mathrm{~ns}$, plasma which has propagated through the coil appears to be focused to a small diameter at $\sim 20 \mathrm{~mm}$ beyond the coil. It can be noted that plasma which has traveled to $20 \mathrm{~mm}$ from the target at $1900 \mathrm{~ns}$ will have passed through the coil at $\sim 950 \mathrm{~ns}$, assuming minimal forward acceleration from interaction with the magnetic field. At this time, the current is increasing, and thus, according to Lenz's law, induced plasma current will be oppositely directed to the coil current. It then follows that the Lorentz interaction of plasma current with the magnetic field will act to drive the plasma towards the axis, thus producing a plasma focusing effect. The focusing plasma is revealed at $1900 \mathrm{~ns}$ because at that time the magnetic field is changing rapidly, leading to high values of inductive heating and light emission.

Figure 4 shows time- and space-resolved optical emission spectra acquired in the time window 1800-2000 ns without and with magnetic field. These spectra are acquired with the image of the spectrometer slit lying along the axis of the coil normal to the target surface. In the field off case (Fig. 4(a)), the plasma has expanded through the coil and the spectral lines are observed out to $25 \mathrm{~mm}$ from the target. As expected, the spectral emission is obscured at the location of the coil. The three $\mathrm{Cu}$ I spectral lines observed in this region 


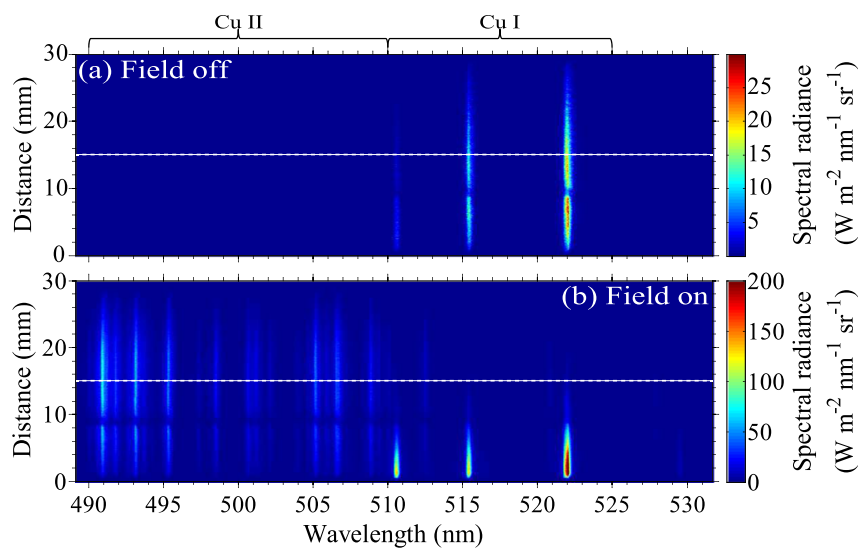

FIG. 4. Space-resolved spectra of the plasma for magnetic field (a) off and (b) on at $1900 \pm 100 \mathrm{~ns}$.

are $\mathrm{Cu} \mathrm{I} 3 \mathrm{~d}^{9} 4 \mathrm{~s}^{2}{ }^{2} \mathrm{D}_{5 / 2}-3 \mathrm{~d}^{10} 4 \mathrm{p}^{2} \mathrm{P}_{3 / 2}(510.55 \mathrm{~nm}), \mathrm{Cu}$ I $3 \mathrm{~d}^{10}$ $4{ }^{2} \mathrm{P}_{1 / 2}-3 \mathrm{~d}^{10} 4 \mathrm{~d}^{2} \mathrm{D}_{3 / 2}(515.32 \mathrm{~nm})$, and $\mathrm{Cu}$ I $3 \mathrm{~d}^{10} 4 \mathrm{p}$ ${ }^{2} \mathrm{P}_{3 / 2}-3 \mathrm{~d}^{10} 4 \mathrm{~d}{ }^{2} \mathrm{D}_{3 / 2,5 / 2}(522.01 \mathrm{~nm}, 521.82 \mathrm{~nm})$. Since the sensitivity of the spectrometer is absolutely calibrated, it is possible to use the emission spectrum to diagnose the plasma conditions as a function of distance from the target. This is done by taking a lineout $(15 \mathrm{~mm}$ from the target) from the space-resolved spectrum and fitting the plot of spectral radiance using the collisional radiative spectral synthesis code PrismSPECT ${ }^{17}$ in non-local thermodynamic equilibrium mode. From Fig. 3, it can be seen that the diameter of the plasma at this position is $\sim 8 \mathrm{~mm}$. The spectrum was fitted with an electron temperature, $T_{e}=0.6 \mathrm{eV}$, atom/ion density, $n_{i}=5 \times 10^{12} \mathrm{~cm}^{-3}$, and a mean ion charge, $Z=0.54$. Spectral calculations for these conditions also reveal that the plasma is optically thin.

The spectrum with magnetic field (Fig. 4(b)) is much richer and includes lines due to $\mathrm{Cu}$ II. The neutral lines are still observed closer to the target, but are not visible on either side of the coil where the inductive heating is more intense and ionized species predominate. The stronger emission lines of ionized $\mathrm{Cu}$ are $\mathrm{Cu}$ II $3 \mathrm{~d}^{9} 4 \mathrm{~d}^{3} \mathrm{G}_{5}-3 \mathrm{~d}^{9} 4 \mathrm{f}^{3} \mathrm{H}_{6}(490.97 \mathrm{~nm})$, $\mathrm{Cu}$ II $3 \mathrm{~d}^{9} 4 \mathrm{~d}^{3} \mathrm{G}_{4}-3 \mathrm{~d}^{9}$ 4f ${ }^{1} \mathrm{H}_{5}(493.17 \mathrm{~nm}), \mathrm{Cu}$ II $3 \mathrm{~d}^{9} 4 \mathrm{~d}$ ${ }^{1} \mathrm{G}_{4}-3 \mathrm{~d}^{9} 4 \mathrm{f}^{3} \mathrm{H}_{5}$ (495.37 nm), and Cu II $3 \mathrm{~d}^{10} 4 \mathrm{~d}^{3} \mathrm{~F}_{4}-3 \mathrm{~d}^{9} 4 \mathrm{f}$ ${ }^{3} \mathrm{G}_{5}(505.18 \mathrm{~nm})$. Fitting the lineout of the spectral radiance at $15 \mathrm{~mm}$ yields $T_{e}=2.1 \mathrm{eV}, n_{i}=3 \times 10^{13} \mathrm{~cm}^{-3}$, and $\mathrm{Z}=2$ for a plasma diameter of $6.5 \mathrm{~mm}$. This spectroscopic analysis shows more than 3-fold increase in plasma temperature, clearly indicating that significant inductive heating has occurred. This conclusion is further reinforced by the presence of $\mathrm{Cu}$ II emission in the region between the target and the coil.

Figure 5 shows the spatial distribution of the deposition per laser pulse, with and without magnetic field, on a glass substrate placed at $\sim 20 \mathrm{~mm}$ from the target $(10 \mathrm{~mm}$ beyond the coil). To make the depositions, the laser was guided between the target and the coil, and the angle of incidence on the target was $45^{\circ}$, making the ablation spot elliptical. The laser energy was increased, so as to maintain the laser fluence of $2 \mathrm{~J} \mathrm{~cm}^{-2}$. The deposition distributions were obtained by using a flatbed transmission scanner to map the optical transmission of films made, using 8000 shots for the field off and 500 shots with the field on, as described by
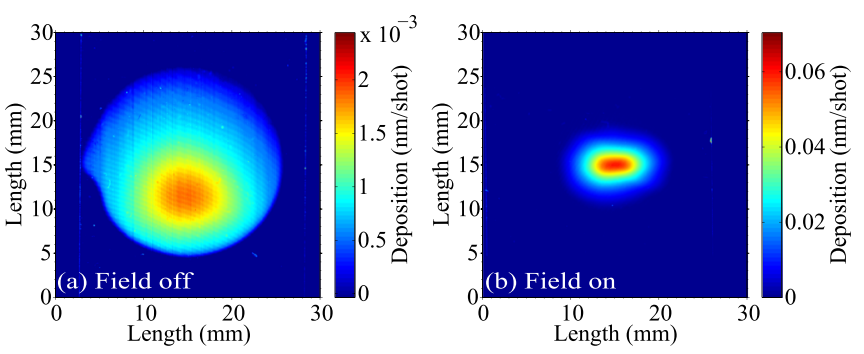

FIG. 5. Spatial distributions of $\mathrm{Cu}$ deposition rate per laser pulse on glass substrates at $10 \mathrm{~mm}$ beyond the induction coil for magnetic field (a) off and (b) on.

Donnelly et al. ${ }^{18}$ The boundary of the deposition in the nofield case (Fig. 5) corresponds to a projection of the coil aperture from the ablation spot. The total number of $\mathrm{Cu}$ atoms deposited per shot is $2.4 \times 10^{13}$. Fitting the deposition profile yields a value of $\sim 2.3$ for the aspect ratio of the ablation plume. ${ }^{16}$ Using this value, and the deposition rate of $2 \times 10^{-3} \mathrm{~nm}$ per laser shot in the center of the profile, gives a value of $\sim 7.5 \times 10^{13}$ for the number of atoms deposited per pulse, ${ }^{13,16}$ if the plume was not skimmed by the coil aperture. The deposition with the field (Fig. 5(b)) was confined to a much smaller area and the maximum deposition rate is increased to $70 \times 10^{-3} \mathrm{~nm}$ per laser shot, which is $35 \times$ higher than without the field. The total number of atoms deposited was $7.8 \times 10^{13}$ which agrees well with the total number of atoms in the ablation plume.

All the observations above show that the application of a strong pulsed magnetic field to a LPP in a vacuum gives rise to a large increase of the optical emission, enhancement of the ionization, and focusing of the plasma transmitted through the central aperture of the coil. These features can be quantitatively understood in terms of heating due to induced currents and the associated MHD response. Analysis of the emission spectrum from just inside the coil at $\sim 700 \mathrm{~ns}$ indicates that $T_{e}=0.5 \mathrm{eV}$ and $n_{i}=7.5 \times 10^{13} \mathrm{~cm}^{-3}$. For this value of $T_{e}$, the Spitzer electrical conductivity ${ }^{19}$ has a value of $\sigma=800 \Omega^{-1} \mathrm{~m}^{-1}$. Since electrical conduction occurs across the magnetic field lines, a value equal to half the Spitzer value, ${ }^{19}$ namely, $400 \Omega^{-1} \mathrm{~m}^{-1}$, is used. The time scale $\tau=\mu_{0} \sigma R^{2}$ for diffusion of the magnetic field across the plasma radius $R \sim 5 \mathrm{~mm}$ has a value of $\sim 13 \mathrm{~ns}$. Since this is much shorter than the period of the discharge, it may be assumed that the external magnetic field fully penetrates the plasma.

The inductive coupling of the magnetic coil and the LPP can be analyzed according to resistive MHD. In that case, the momentum equation ${ }^{20}$ is

$$
\rho \frac{d \boldsymbol{u}}{d t}=\boldsymbol{j} \times \boldsymbol{B}-\nabla P,
$$

where $\boldsymbol{u}$ is the local plasma velocity, $\boldsymbol{j}$ is the current density, and $P$ is the plasma pressure. The current density in Eq. (1) is given by the generalized form of Ohm's law ${ }^{20}$

$$
\boldsymbol{E}+\boldsymbol{u} \times \boldsymbol{B}=\frac{\boldsymbol{j}}{\sigma}+\frac{1}{n_{e} e}(\boldsymbol{j} \times \boldsymbol{B}-\nabla P) .
$$

For the plasma considered here, the ambipolar electric field arising from the gradient of electron pressure is small 
compared to the induced field and $\boldsymbol{u} \times \boldsymbol{B}$. It can also be noted that for the cylindrical plasma in the region of the coil, the induced current $\boldsymbol{j}$ is constrained to flow in the azimuthal direction. Thus, $\boldsymbol{j}$ can be written as $\boldsymbol{j}=\sigma(\boldsymbol{E}+\boldsymbol{u} \times \boldsymbol{B})$, and the Hall term $\boldsymbol{j} \times \boldsymbol{B} / n_{e} e$ corresponds to a field in the radial direction and gives rise to an $\mathbf{E} \times \mathbf{B}$ drift. This drift would be manifested as a rotation of the plasma about the axis of symmetry.

The rate of change of magnetic field has a maximum value of $5.9 \times 10^{5} \mathrm{~T} \mathrm{~s}^{-1}$ at $\sim 700 \mathrm{~ns}$ which gives rise to an azimuthal electric field of $\sim 1500 \mathrm{~V} \mathrm{~m}^{-1}$ near the edge of the aperture in the coil. Since $\boldsymbol{B}$ goes to zero at this time, there is no contribution from the $\boldsymbol{u} \times \boldsymbol{B}$ term in Eq. (2). The power input to the plasma due to resistive heating is given by $\sigma E^{2}$ and will have a value of $8.8 \times 10^{8} \mathrm{~W} \mathrm{~m}^{-3}$. Thus, in a time interval of $200 \mathrm{~ns}$, the energy input due to resistive heating is $180 \mathrm{~J} \mathrm{~m}^{-3}$ or $\sim 15.0 \mathrm{eV}$ per atom/ion for a plasma density of $7.5 \times 10^{19}$ atom/ion $\mathrm{m}^{-3}$. This compares well with the value of internal energy of $23 \mathrm{eV} /$ atom for a plasma at $T_{e}=1.5 \mathrm{eV}$.

Equation (1) can be used to estimate the radial forces exerted on the plasma column as it passed through the high magnetic field in the region of the coil aperture. As mentioned above, focusing of the plasma is very evident in Fig. 3 at $1900 \mathrm{~ns}$. Plasma at $20 \mathrm{~mm}$ from the target at $1900 \mathrm{~ns}$ will have passed through the coil aperture at $950 \mathrm{~ns}$. At that time, the induced electric field near the edge of the coil aperture is $850 \mathrm{~V} \mathrm{~m}^{-1}$. The $\boldsymbol{u} \times \boldsymbol{B}$ term in the Ohm's law equation (Eq. (2)) gives an electric field of $500 \mathrm{~V} \mathrm{~m}^{-1}$. Thus, the induced azimuthal current density near the edge of the aperture is $j_{\phi} \sim 2.5 \times 10^{6} \mathrm{~A} \mathrm{~m}^{-2}$. For a plasma density of $6.5 \times 10^{-6} \mathrm{~kg} \mathrm{~m}^{-3}$ estimated via PrismSPECT fits, the $\boldsymbol{j} \times \boldsymbol{B}$ force gives rise to a radial acceleration of $\sim 3.8 \times 10^{10} \mathrm{~m} \mathrm{~s}^{-2}$ directed towards the axis. The gradient of the plasma pressure in Eq. (1) is much less than this value. It takes $\sim 200 \mathrm{~ns}$ for a plasma element to traverse the region of high field near the plasma of the coil, and in that time the change in the radial velocity is $-7.6 \times 10^{3} \mathrm{~m} \mathrm{~s}^{-1}$. Considering that plasma reaching the edge of the coil at $950 \mathrm{~ns}$ has an outward directed radial velocity of $\sim 5 \times 10^{3} \mathrm{~m} \mathrm{~s}^{-1}$, the plasma will leave the high field region with $d r / d t=-2.6 \times 10^{3} \mathrm{~m} \mathrm{~s}^{-1}$. Thus, we can expect that the plasma column will be brought to a focus at a distance in front of the coil of the same order as the radius of the coil aperture. This is indeed what is observed at $1900 \mathrm{~ns}$, as shown in Fig. 3 with the focused plasma made visible by the intense inductive heating at that time.
Inductive energy coupling to a laser produced copper plasma in a vacuum has been demonstrated. Heating and focusing of the plasma plume are apparent in the optical images, emission spectroscopy, and spatial distribution of copper deposition. The measured increase in plasma temperature was consistent with a calculation of the inductive heating. The focusing of the plasma transmitted through the coil aperture was estimated according to resistive MHD, and agreed with experiment. However, a comprehensive MHD simulation should provide a more detailed description of plasma heating and focusing mechanisms. There is scope to optimize the effect for particular applications by varying synchronization of the laser ablation and pulsed field and exploring other magnetic coil configurations. This technique of inductive coupling to a LPP will open up new possibilities in LIBS and PLD.

${ }^{1}$ R. Jordan, D. Cole, and J. G. Lunney, Appl. Surf. Sci. 109-110, 403 (1997).

${ }^{2}$ T. Kobayashi, H. Akiyoshi, and M. Tachiki, Appl. Surf. Sci. 197-198, 294 (2002).

${ }^{3}$ C. Pagano, A. A. Goncharov, and J. G. Lunney, Rev. Sci. Instrum. 83, 02B701 (2012).

${ }^{4}$ L. Dirnberger, P. E. Dyer, S. R. Farrar, and P. H. Key, Appl. Phys. A 59, 311 (1994).

${ }^{5}$ S. S. Harilal, M. S. Tillack, B. O'Shay, C. V. Bindhu, and F. Najmabadu, Phys. Rev. E 69, 0264131 (2004).

${ }^{6}$ C. Pagano, S. Hafeez, and J. G. Lunney, J. Phys. D: Appl. Phys. 42, 1552051 (2009).

${ }^{7}$ K. J. Mason and J. M. Goldberg, Anal. Chem. 59, 1250 (1987).

${ }^{8}$ S. Suckewer, C. H. Skinner, H. Milchberg, C. Keane, and D. Voorhees, Phys. Rev. Lett. 55, 1753 (1985).

${ }^{9}$ T. Pisarczyk, A. Farynski, H. Fiedorowicz, P. Gogolewski, M. Kusnierz, J. Makowski, R. Miklaszewski, M. Mroczkowski, P. Parys, and M. Szczurek, Laser Part. Beams 10, 767 (1992).

${ }^{10}$ C. S. Wong and S. Lee, Rev. Sci. Instrum. 55, 1125 (1984).

${ }^{11}$ I. Tobin, L. Juschkin, Y. Sidelnikov, F. O'Reilly, P. Sheridan, E. Sokell, and J. G. Lunney, Appl. Phys. Lett. 102, 203504 (2013).

${ }^{12} \mathrm{P}$. Blackborow, D. Gustafson, D. Smith, M. Besen, S. Horne, R. D'Agostino, Y. Minami, and G. Denbeaux, Proc. SPIE 6517, 1 (2007).

${ }^{13}$ B. Doggett and J. G. Lunney, J. Appl. Phys. 105, 033306 (2009).

${ }^{14}$ E. Sterling and J. G. Lunney, AIP Conf. Proc. 1278, 567 (2010).

${ }^{15}$ See http://www.comsol.com/ for COMSOL (2016).

${ }^{16}$ S. I. Anisimov, D. Bauerle, and B. S. Luk'yanchuk, Phys. Rev. B 48, 12076 (1993).

${ }^{17}$ See http://www.prism-cs.com/ for PrismSPECT (2016).

${ }^{18}$ T. Donnelly, J. G. Lunney, S. Amoruso, R. Bruzzese, X. Wang, and X. Ni, J. Appl. Phys. 108, 043309 (2010).

${ }^{19}$ F. F. Chen, Introduction to Plasma Physics (Springer US, 1974), p. 183.

${ }^{20}$ J. Sanderson and T. Boyd, The Physics of Plasmas (Cambridge University Press, 2003), pp. 48-90. 